\title{
Fontes e concentrações de águas de fumaça na germinação de sementes e no vigor de plântulas de tomate
}

\author{
Yêda Maria BC Arruda ${ }^{1}$; Isolde Dorothea K Ferraz ${ }^{2}$; Maria Cristina de F e Albuquerque ${ }^{3}$ \\ ${ }^{1}$ UFAM-Depto. Biologia, Av. General Rodrigo Octávio Jordão Ramos 3000,69080-900 Manaus-AM; yedaarruda@gmail.com; ${ }^{2}$ INPA- \\ CBio, C. Postal 2223, 69080-971 Manaus-AM; iferraz@inpa.gov.br; ${ }^{3}$ UFMT-FAMEV, Depto. Fitotecnia e Fitossanidade, 78090-600 \\ Cuiabá-MT; albuquerquemcfa@gmail.com
}

\section{RESUMO}

A fumaça e a fumaça diluída em água, conhecida como água de fumaça, podem ser utilizadas como estimuladores tanto da germinação das sementes quanto do vigor das plântulas de várias espécies silvestres e cultivadas. O objetivo neste trabalho foi verificar a eficácia de águas de fumaça, oriundas de diferentes matérias-primas e concentrações, na germinação de sementes e vigor de plântulas de tomate. Foram usadas 10 matérias-primas e para cada uma foram testados um controle (água destilada) e oito concentrações da água de fumaça (1:5.000, 1:2.500, 1:1.000, 1:100, 1:50, 1:25, 1:10 e 1:2,5 $\mathrm{v}: \mathrm{v})$, constituindo nove tratamentos, que foram repetidos oito vezes e distribuídos seguindo o delineamento experimental inteiramente casualizado. Os testes germinativos foram conduzidos no laboratório e as variáveis foram avaliadas após cinco dias da semeadura. Com a exceção de uma fonte, todas as matérias-primas testadas causaram, com a maior concentração testada $(1: 2,5)$, inibição na germinação das sementes de tomate. Efeito estimulador foi observado principalmente em águas de fumaça produzidas com matérias-primas de folhas de palmeira (Euterpe precatoria) e de milho (Zea mays), com madeira leve de uma espécie pioneira (Cecropia palmata); serragem de espécies madeireiras amazônicas (Dinizia excelsa, Sextonia sp., Licania spp. e Aniba spp.) e de papel de germinação. Esse efeito foi mais expressivo no comprimento de raiz e no índice de vigor, nas concentrações de 1:25 a 1:5.000, de acordo com a fonte; a concentração de 1:25 sobressaiu na maioria das matérias-primas testadas.

Palavras-chave: Lycopersicon esculentum, fumaça de origem vegetal; butenolídio; índice de vigor.

\begin{abstract}
Sources and concentrations of smoke-water on the seed germination and seedling vigor of tomato
\end{abstract}

Smoke and smoke diluted in water, known as smoke-water, can be used to promote both seed germination and seedling vigor of several wild and cultivated species. The objective of this study was to assess the effectiveness of smoke-water produced with different sources (raw materials) and concentrations on seed germination and seedling vigor of tomato. Smoke-water was produced with ten different raw materials, for each was done a control (distilled water) and eight concentrations with smoke-water (1:5,000, 1:2,500, 1:1,000, 1:100, $1: 50,1: 25,1: 10$ e 1:2.5 v:v), making up nine treatments repeated eight times and distributed following a completely randomized design. Germination was assessed in the laboratory and the variables were evaluated five days after sowing. With the exception of one source, all tested raw materials showed, with the highest concentration tested (1:2.5), inhibitory effect on the germination of the tomato seeds. Promoting effect was observed mainly in smoke-waters produced from raw materials of palm leaves (Euterpe precatoria) and corn leaves (Zea mays), of light wood from a pioneer species (Cecropia palmata), sawdust of amazonian timber species (Dinizia excelsa; Sextonia sp.; Licania spp.; Aniba spp.) and of germination paper. The promoting effect was more significant on root length and on vigor index with concentrations between 1:25 and 1:5,000, in accordance with the source. The dilution of 1:25 excelled in most of the tested raw materials.

Keywords: Lycopersicon esculentum, plant derived smoke, butenolide, vigour index.

\section{(Recebido para publicação em 16 de setembro de 2011; aceito em 28 de maio de 2012) (Received on September 16, 2011; accepted on May 28, 2012)}

\begin{abstract}
A fumaça de origem vegetal, solúvel em água, pode aumentar a germinação das sementes (Crosti et al., 2006), superar a dormência (Brown \& Van Staden, 1997; Jusaitis et al., 2004) e aumentar o vigor das plântulas (Sparg et al., 2005; Van Staden et al., 2006). Água de fumaça é produzida pelo borbulho de fumaça em água e a solução pode ser aplicada para diversos fins.

Várias espécies com importância ecológica e econômica já foram alvo de estudos sobre o efeito da fumaça. Pesquisas com espécies nativas ocorrentes em vários ecossistemas foram
\end{abstract}

realizadas nos Estados Unidos (Keeley \& Fotheringham, 1998), na Austrália (Dixon et al., 1995; Roche et al., 1998) e na África do Sul(Brown, 1993; Brown et al., 2003). Sementes de espécies economicamente viáveis do setor da medicina popular (Sparg et al., 2005; Kulkarni et al., 2006a, 2007a) e do setor produtivo, apresentaram também respostas positivas a diversos tipos de aplicação da fumaça, como alface (Jäger et al., 1996), arroz (Kulkarni et al., 2006b), quiabo, tomate (Kulkarni et al., 2007b; Van Staden et al., 2007) e milho (Sparg et al., 2006; Van Staden et al., 2006). Para espécies de olerícolas como o tomate, o vigor da plântula é muito importante por garantir mudas vigorosas de tamanho uniforme.

O componente biologicamente ativo e comprovado é o butenolídio (3-metil-2H-furo[2,3-c]piran-2-ose) identificado após queima da celulose (Flematti et al., 2004) e combustão de material vegetal (Van Staden et al., 2004). Em escala laboratorial, a água de fumaça é preparada, geralmente, com papel de germinação, ou pela queima de espécies herbáceas nativas, por exemplo Themeda triandra e Passerina vulgaris 
(Jain \& Van Staden, 2006). Porém, outros materiais de origem vegetal foram testados para a sua produção, como por exemplo, folhas de angiospermas e gimnospermas (Jäger et al., 1996). $\mathrm{O}$ efeito parece variar em função da espécie (Jäger et al., 1996), do tipo de material (galho ou folha) usado na combustão, o seu estado (seco e/ou fresco), bem como a concentração de fumaça na água (Brown \& Van Staden, 1997; Minorsky, 2000).

Desta forma, o objetivo neste trabalho foi verificar a eficácia de águas de fumaça oriundas de diferentes matérias-primas e concentrações na germinação de sementes e vigor de plântulas de tomate.

\section{MATERIAL E MÉTODOS}

Os experimentos foram realizados em laboratório do INPA em Manaus-AM, no ano de 2007, com matéria-prima coletada de diferentes espécies da região amazônica.

A preparação dos extratos de fumaça seguiu a metodologia desenvolvida por Farley (2005). Dez fontes (matérias-primas) foram selecionadas e agrupadas conforme a sua origem, em grupo das folhas: folha da palmeira de açaí (Euterpe precatoria) (FP), da imbaúba (Cecropia palmata) (FI), da palha da espiga do milho (Zea mays) (FM); grupo das madeiras: de imbaúba $(C$. palmata) (MI), serragem das madeiras de louro-gamela (Sextonia sp.), de angelim-vermelho (Dinizia excelsa) (MSI) e de várias espécies denominadas popularmente louro (Licania spp. / Aniba spp.) (MSII); grupo dos resíduos: sobras do ouriço da castanha-da-amazônia (Bertholletia excelsa) (RC), da casca da semente de andiroba (Carapa guianensis) (RSA), da valva do fruto de andiroba (C. guianensis) (RFA); além do papel de germinação fino tipo Germitest $^{\circledR}$ (PG).

As matérias-primas foram previamente secas em estufa a $75^{\circ} \mathrm{C}$ por até 24 horas e, em seguida, queimadas totalmente e a fumaça produzida foi levada por sucção para um kitassato contendo água destilada na proporção de $10 \mathrm{~L}$ para $6 \mathrm{~kg}$ de matéria-prima, seguindo a metodologia de Flematti et al. (2004). A temperatura da solução durante a queima atingiu no máximo $61^{\circ} \mathrm{C}$. Os extratos concentrados, após resfriamento e filtragem com papel de filtro, foram armazenados a $15^{\circ} \mathrm{C}\left( \pm 2^{\circ} \mathrm{C}\right)$ e usados por no máximo três meses.

Para cada matéria-prima foram testados um controle (água destilada) e oito concentrações da água de fumaça em água destilada (1:5.000, 1:2.500, $1: 1.000,1: 100,1: 50,1: 25,1: 10$ e $1: 2,5$ $\mathrm{v}: \mathrm{v})$, constituindo nove tratamentos, que foram repetidos oito vezes e distribuídos seguindo o delineamento experimental inteiramente casualizado.

A ação dos extratos foi testada em sementes de tomate (Lycopersicon esculentum) cultivar Santa Adélia Super, seguindo a metodologia aplicada por Jain \& Van Staden (2006) com a mesma espécie. A semeadura foi realizada no escuro e as sementes mantidas nesta condição por cinco dias a $25^{\circ} \mathrm{C} \pm 2{ }^{\circ} \mathrm{C}$, em caixas de plástico tipo "gerbox", sobre quatro camadas de papel de germinação fino, tipo Germitest ${ }^{\circledR}$.

Foram estudados o porcentual de sementes germinadas (considerando raiz primária $\geq 2 \mathrm{~mm}$ independente se as raízes tinham ou não pelos radiculares), porcentual de plântulas normais e anormais, comprimento da raiz primária e do hipocótilo ( $\mathrm{mm})$, comprimento total (mm) e o quociente do índice de vigor (QIV). O índice de vigor (IV) foi calculado de acordo com Dhindwal et al. (1991): IV = [comprimento do hipocótilo $(\mathrm{mm})+$ comprimento da raiz $(\mathrm{mm})]$ $\mathrm{x}$ porcentagem final da germinação. $\mathrm{O}$ QIV é a razão do IV do tratamento pelo IV do controle.

A plântula de tomate foi considerada normal (Figura 1a) quando apresentou alongamento da raiz primária e do hipocótilo e plântula anormal (Figura 1b) quando apresentou emergência da raiz primária sem o seu alongamento, conforme também observado por Jain \& Van Staden (2006).

A análise de variância foi realizada para cada matéria-prima e variável, e quando houve significância para tratamentos $(p(F) \leq 0,05)$ as médias foram comparadas pelo teste de Tukey ( $\alpha=$ $0,05)$. Previamente, os dados foram submetidos aos testes de Shapiro-Wilk (teste de normalidade dos resíduos) e de Levene (teste de homogeneidade entre variâncias), e quando necessário, aqueles referentes à porcentagem de germinação foram transformados em arco seno $\sqrt{\mathrm{x} / 100}$, e os demais em $\log _{10}$ $(\mathrm{x}+1)$. Para todas as análises estatísticas foi usado o programa PROPHET 5.0.

\section{RESULTADOS E DISCUSSÃO}

Na solução mais concentrada, 1:2,5, a água de fumaça, de todas as matérias -primas, exceto MSII, reduziu significativamente o porcentual de germinação em comparação ao controle (água destilada) (Figura 2), e na concentração de 1:10 esse efeito só foi verificado com quatro extratos: folha da palmeira de açaí, palha da espiga do milho, casca da semente de andiroba e valva do fruto de andiroba. Entre as anormalidades nas plântulas, observou-se principalmente ausência de pelos radiculares (Figura 1), fenômeno também descrito por Jain \& Van Staden (2006).

Efeito inibidor da água de fumaça tem sido verificado em outras espécies e, da mesma forma como observado na presente pesquisa, a concentração nociva depende da espécie utilizada como matéria-prima. Em milho a concentração $1: 250$, oriundo da queima de Themeda triandra, já foi limitante ao crescimento da raiz (Sparg et al., 2006). Com a queima de "fynbos", uma vegetação típica da África do Sul, sementes de Senecio grandiflorus e Restio similis apresentaram inibição da germinação na concentração 1:10 e sementes de Syncarpha vestia na concentração 1:2 (Brown, 1993).

A água de fumaça contém, além da substância ativa, outros produtos e alguns destes podem, em concentrações altas como 1:1.000, ser responsáveis pelo decréscimo no vigor (Van Staden et al., 2004).

Nas concentrações maiores ou igual a 1:25, a germinação (sem distinção entre plântulas normais e anormais) foi superior a $90 \%$, sem diferenças significativas entre as concentrações, independente da matéria-prima (Figura 2). Diversos outros estudos, também não apresentaram efeito da água de fumaça quando analisada somente a porcenta- 

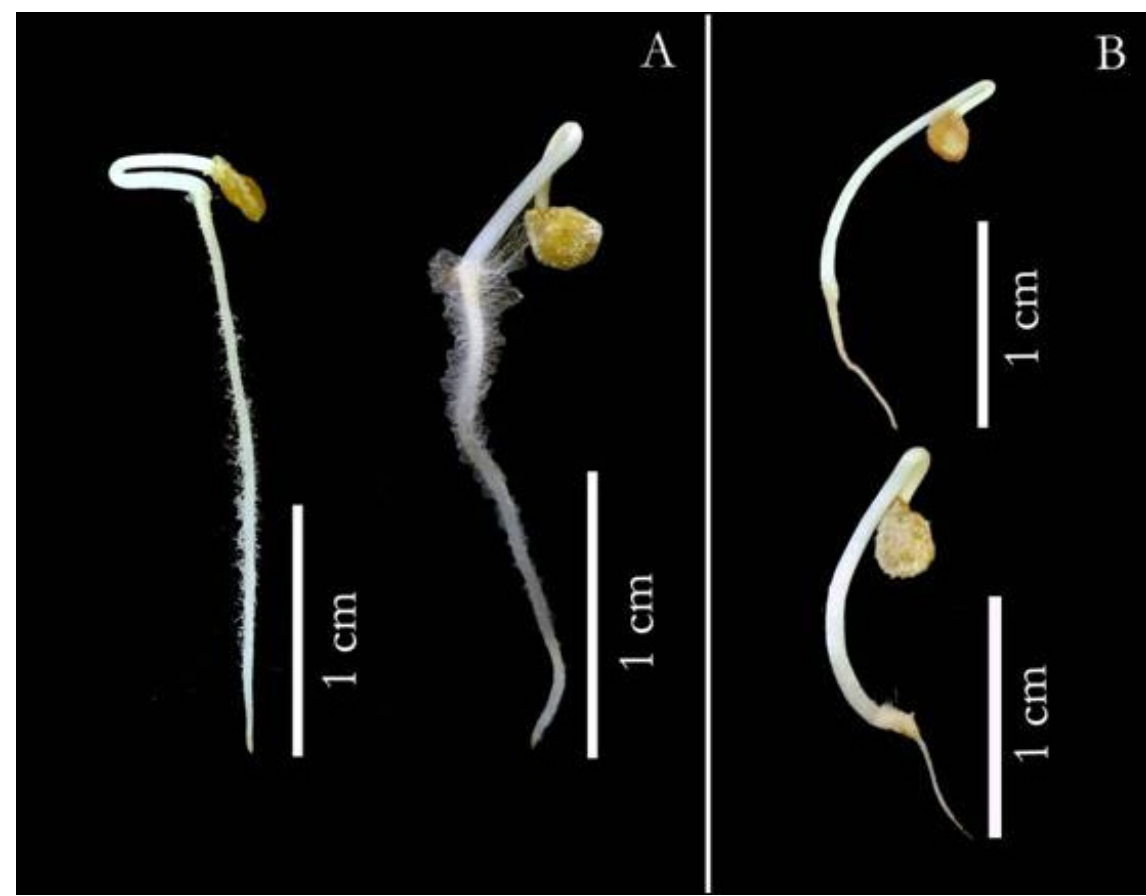

Figura 1. Morfologia das plântulas de tomate, desenvolvidas no escuro a $25 \pm 2^{\circ} \mathrm{C}$, cinco dias após semeadura. (A) plântulas normais e (B) plântulas anormais (morphology of the tomato seedlings, five days after sowing and developed in the darkness $\left(25 \pm 2^{\circ} \mathrm{C}\right)$ : (A) normal seedlings and (B) abnormal seedlings). Manaus, INPA, 2009.

gem de germinação (Sparg et al., 2005; Merritt et al., 2006; Kulkarni et al., 2006a; Kulkarni et al., 2007a, 2007c; Stevens et al., 2007).

Avaliando as plântulas normais registrou-se efeito significativo, dependendo da matéria-prima utilizada na produção da água de fumaça, bem como da concentração testada (Figura 2). O efeito positivo foi registrado com duas matérias-primas do grupo das folhas (FP: $1: 25$ a 1:2500; FM: 1:50 e 1:100), com todas do grupo das madeiras (MI: 1:25 e 1:50; MSI: 1:25 a 1:100; MSII: $1: 10$ e 1:25) e com duas do grupo dos resíduos (RSA: 1:25; RFA: 1:25), além da água de fumaça produzida com $P G$ (1:25). Os resultados confirmaram, para o tomate, que a água de fumaça pode reduzir anormalidades das plântulas, como descrito para as espécies de Erica spp. (Brown, 1993). A redução de anormalidade das plântulas do tomate também foi observada por Jain \& Van Staden (2006) ao testar o agente bioativo da água de fumaça (butenolídio).

O crescimento da raiz primária foi significativamente maior utilizando como matéria-prima papel de germinação (PG) na concentração 1:25; folhas de palmeira (FP: 1:100 e 1:2500) e da palha da espiga do milho (FM: 1:50 e $1: 100)$ e com todas as madeiras (MI: 1:25 e 1:50; MSI: 1:100; MSII: 1:10 e 1:25). Em todos os tratamentos mencionados, o crescimento foi de duas a três vezes maior quando comparado com seus respectivos controles (Figura 3). Sparg et al. (2005) observaram que o efeito da fumaça pode ser visualizado melhor nos estágios pós-germinativos, como no comprimento da raiz e do caule, peso e sobrevivência das plântulas.

Em geral, as águas de fumaça influenciaram menos o crescimento do hipocótilo (Figura 3). Aumento significativo foi observado somente nas águas de fumaça produzidas com FP (concentração 1:2500), MI (1:50) e MSI (1:100): até 1,5 vezes maior do que o controle.

O comprimento total das plântulas teve comportamento similar ao comprimento da raiz, sendo até duas vezes maior do que o obtido com água destilada quando se utilizou água de fumaça oriunda da queima de papel de germinação (PG: 1:25), da palha da espiga do milho (FM: 1:50) e de todas as madeiras (MI:1:50; MSI: 1:100; MSII: $1: 10$ e $1: 25)$.

Comparando as três medidas de comprimento (raiz, hipocótilo e total), os resultados deste estudo mostraram claramente que a ação estimuladora da água de fumaça foi mais evidente no sistema radicular, como também observado por Van Staden et al. (2006) na mesma espécie. O comprimento total da plântula de tomate foi menos eficiente para detectar o efeito estimulador da água de fumaça por ser o somatório de uma variável sensível (raiz) com uma menos sensível (hipocótilo).

$\mathrm{O}$ quociente do índice de vigor indica quantas vezes um determinado tratamento foi superior ou inferior ao controle. Quando se analisou esse quociente (Figura 4), destacaram-se FP (1:2500) e MSI (1:100) como as matérias-primas mais eficientes no efeito estimulador da água de fumaça, pois duplicaram o vigor em relação ao controle. $\mathrm{O}$ aumento do vigor de 1,5 a 2,4 vezes em relação ao controle (corresponde ao valor 1 na figura) foi observado com: PG (1:25), MI (1:50) e FP (1:5000).

Ficou evidente que, conforme demonstrado para outras espécies (Sparg et al., 2005, 2006; Van Staden et al., 2006; Kulkarni et al., 2006b, 2007a, 2007c), a ação da água de fumaça no tomate também não pode ser analisada somente pela porcentagem de germinação das sementes, sendo mais eficiente usar atributos de vigor como o desenvolvimento da plântula.

Destacaram-se duas variáveis com alta sensibilidade à água de fumaça. A variável "plântula normal" foi a mais robusta na determinação do estímulo positivo da água de fumaça, acusando diferença significativa entre o controle e 17 concentrações. A segunda variável foi o "comprimento da raiz" que acusou diferença entre o controle e 10 concentrações. Esta última foi vantajosa pela maior facilidade e objetividade na sua avaliação.

As concentrações que causaram efeito positivo e significativo nas diversas variáveis estão na faixa entre 1:10 e 1:2.500. Entretanto o efeito estimulador manifestou-se prioritariamente nas concentrações $1: 25$ a 1:100, indicando uma faixa de maior influência positiva das águas de fumaça. Além disso, as águas de fumaça PG, FP, MI e MSI apresentaram mais resultados positivos em todas 


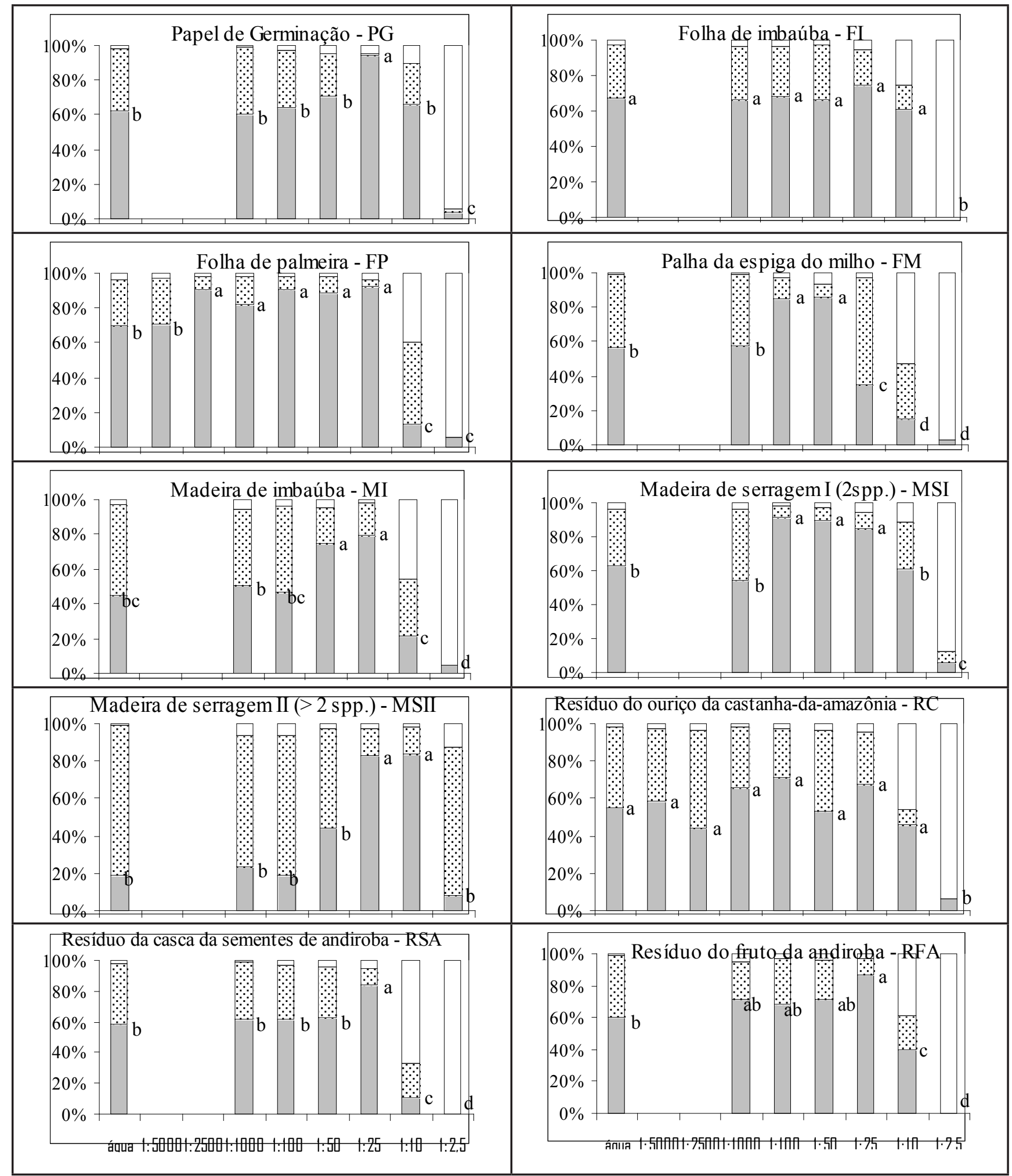

Figura 2. Percentuais de plântulas normais $(\square)$, anormais (罒) e sementes não germinadas $(\square)$ de tomate, no escuro a $25 \pm 2^{\circ} \mathrm{C}$, cinco dias após a semeadura sob diferentes concentrações da água de fumaça produzidas pela queima de papel de germinação (PG); folha de palmeira (FP); folha de imbaúba (FI); palha da espiga de milho (FM); madeira de imbaúba (MI); serragem das madeiras de louro-gamela e angelimvermelho (MSI); e de várias espécies denominadas popularmente louro (MSII); resíduo do ouriço da castanha-da-amazônia (RC); resíduo da casca da semente de andiroba (RSA); resíduo do fruto de andiroba (RFA). Médias das plântulas normais seguidas pela mesma letra não diferem entre si pelo teste de Tukey ( $>>0,05)$ (percentage of normal seedlings $(\square)$, abnormal (国) and no germinated seeds ( $\square$ ) of tomato, in the darkness at $25 \pm 2{ }^{\circ} \mathrm{C}$, five days after sowing under different concentrations of the smoke-water produced by burns of germination paper (PG); palm tree leaf (FP); imbaúba leaf (FI); corn leaf (FM); imbaúba wood (MI); sawdust of louro-gamela and angelim-vermelho wood (MSI); and more popular species commonly called louro (MSII); residue of the Brazil nut (RC); residue of the tegument andiroba seed (RSA); residue of the andiroba fruit (RFA). Averages of normal plantlets followed by equal letters did not differ at $5 \%$ probability by Tukey test). Manaus, INPA, 2009. 


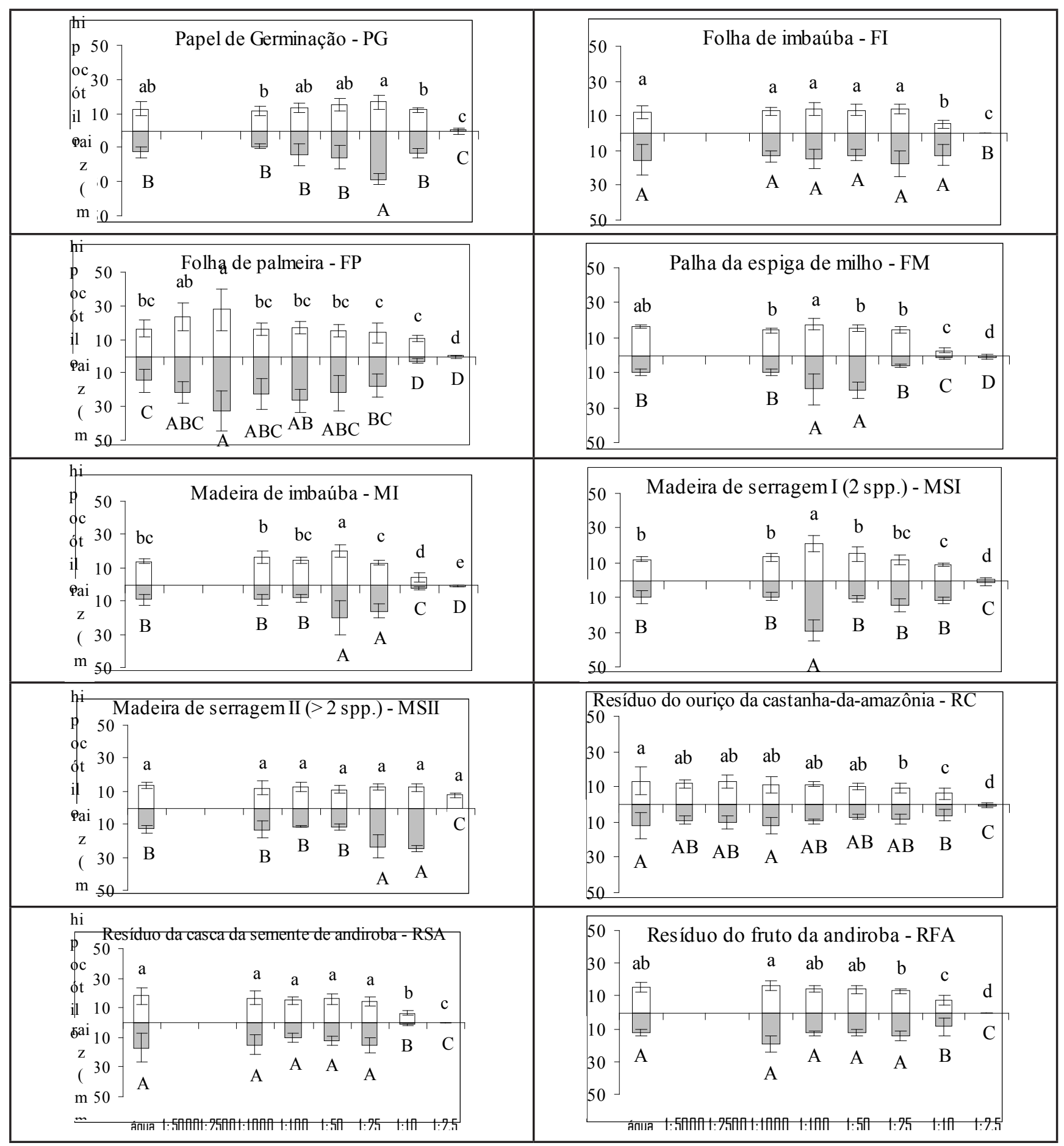

Figura 3. Comprimento da raiz $(\square)$ e do hipocótilo $(\square)$ das plântulas de tomate, cinco dias após a semeadura e o desenvolvimento no escuro $\left(25 \pm 2^{\circ} \mathrm{C}\right)$ sob diferentes concentrações da água de fumaça produzidas pela queima de: papel de germinação (PG); folha de palmeira (FP); folha de imbaúba (FI); palha da espiga de milho (FM); madeira de imbaúba (MI); serragem das madeiras de louro-gamela e angelim-vermelho (MSI); e de várias espécies denominadas popularmente louro (MSII); resíduo do ouriço da castanha-da-amazônia (RC); resíduo da casca da semente de andiroba (RSA); resíduo do fruto de andiroba (RFA). Médias dos comprimentos seguidas pela mesma letra (maiúscula para raiz e minúscula para hipocótilo) não diferem entre si pelo teste de Tukey (p>0,05) (length of the root ( $\square$ ) and of the hypocotyl ( $\square$ ) of the tomato seedlings, five days after the sowing and the development in the darkness $\left(25 \pm 2^{\circ} \mathrm{C}\right)$ under different concentrations of the smokewater produced by burns of germination paper (PG); palm tree leaf (FP); imbaúba leaf (FI); corn leaf (FM); imbaúba wood (MI); sawdust of louro-gamela and angelim-vermelho wood (MSI); and more popular species commonly called louro (MSII); residue of the Brazil nut (RC); residue of the tegument of andiroba seed (RSA); residue of the andiroba fruit (RFA). Averages of the lenghts followed by equal letters (uppercase for roots and lowercase for hypocotyl) did not differ at 5\% probability by Tukey test). Manaus, INPA, 2009. 


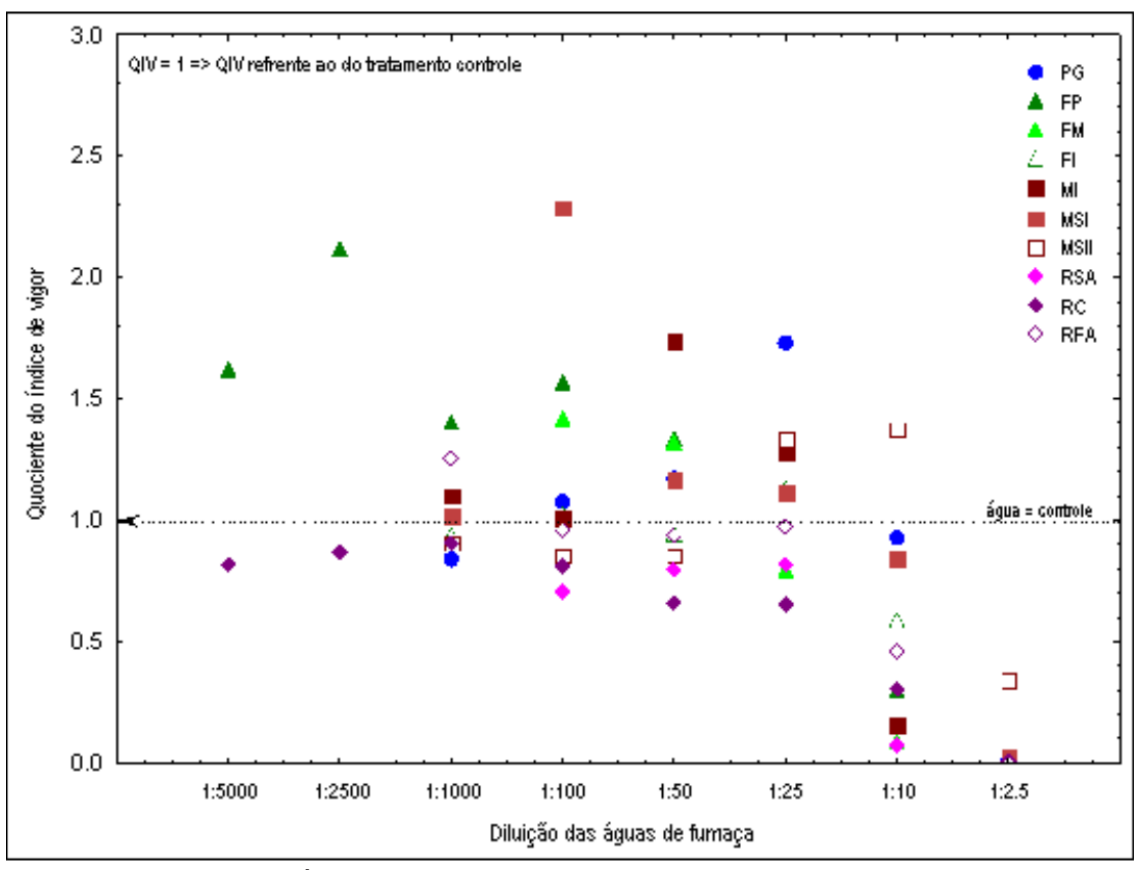

Figura 4. Quociente do Índice de Vigor das plântulas de tomate, cinco dias após a semeadura e o desenvolvimento no escuro $\left(25 \pm 2^{\circ} \mathrm{C}\right)$ sob diferentes concentrações da água de fumaça produzidas pela queima de papel de germinação (PG); folha de palmeira (FP); folha de imbaúba (FI); palha da espiga de milho (FM); madeira de imbaúba (MI); serragem das madeiras de louro-gamela e angelim-vermelho (MSI); e de várias espécies denominadas popularmente louro (MSII); resíduo do ouriço da castanha-da-amazônia (RC); resíduo da casca da semente de andiroba (RSA); resíduo do fruto de andiroba (RFA) (quotient of vigor index of the tomato seedlings, five days after the sowing and the development in the darkness $\left(25 \pm 2^{\circ} \mathrm{C}\right)$ under different concentrations of the smoke-water produced by burns of germination paper (PG); palm tree leaf (FP); imbaúba leaf (FI); corn leaf (FM); imbaúba wood (MI); sawdust of louro-gamela and angelim-vermelho wood (MSI); and more popular species commonly called louro (MSII); residue of the Brazil nut (RC); residue of the tegument andiroba seed (RSA); residue of the andiroba fruit (RFA). Manaus, INPA, 2009.

as variáveis. Contudo, duas matérias-primas, RFA e FP, apresentaram efeito estimulador em soluções mais diluídas (1:1.000 e 1:2.500, respectivamente).

Todas as matérias-primas para a produção da água de fumaça, nas concentrações mais altas testadas, 1:2,5, $1: 10$ e $1: 25$, apresentam efeito inibidor sobre a germinação das sementes e/ ou desenvolvimento das plântulas de tomate. O efeito estimulador da água de fumaça, com ação mais expressiva nos eventos pós-germinativos (comprimento da raiz e índice de vigor das plântulas de tomate), ocorreu para seis das dez matérias primas testadas: três do grupo das madeiras (MI, MSI e MSII), duas águas do grupo das folhas (FP e FM), além do papel de germinação; as diluições com maior influência positiva foram 1:25, $1: 50$ e $1: 100$.

\section{REFERÊNCIAS}

BROWN NAC. 1993. Promotion of germination of fynbos seeds by plant-derived smoke. New Phytol. 123:575-583.

BROWN NAC; VAN STADEN J. 1997. Smoke as a germination cue: a review. Plant Growth Regulation 22: 115-124.

BROWN NAC; VAN STADEN J; DAWS MI. 2003. A summary of patterns in the seed germination response to smoke in plants from the Cape Floral Region. In: SMITH RD; DICKIE JB; LININGTON SH; PRITCHARD HW; PROBERT RJ (eds). Seed conservation: turning science into practice. London: The Royal Botanical Gardens. p. 564-574.

CROSTI R; LADD PG; DIXON KW; PIOTTO B. 2006. Post-fire germination: the effect of smoke on seeds of selected species from the central Mediterranean basin. Forest Ecology and Management 221: 306-312.

DHINDWALAS; LATHER BPS; SINGH J. 1991. Efficacy of seed treatment on germination, seedling emergence and vigour of cotton (Gossypium hirsutum) genotypes. Seed
Research 19: 59-61.

DIXON KW; ROCHE S; PATE JS. 1995. The promotive effect of smoke derived from burnt native vegetation on seed germination of Western Australian plants. Oecologia 101: 185-192.

FARLEY GJ. 2005. The implications of a reproducible method for making smoke water on seed dormancy studies. In: INTERNATIONAL WORKSHOP ON SEEDS GERMINATING NEW IDEAS, $8^{\text {th }}$. Abstracts... Brisbane: Queensland Australia. p. 130.

FLEMATTI GR; GHISALBERTI EL; DIXON KE; TRENGOVE RD. 2004. A compound from smoke that promotes seed germination. Science 305: 977.

JÄGER AK; LIGHT ME; VAN STADEN J. 1996. Effects of source of plant material and temperature on the production of smoke extracts that promote germination of lightsensitive lettuce seeds. Environmental and Experimental Botany 36: 421-429.

JAIN N; VAN STADEN J. 2006. A smokederived butenolide improves early growth of tomato seedlings. Plant Growth Regulation 50: $139-148$.

JUSAITIS M; POLOMKA L; SORENSEN B. 2004. Habitat specificity, seed germination and experimental translocation of the endangered herb Brachycome muelleri (Asteraceae). Biological Conservation 116: 251-266.

KEELEY JE; FOTHERINGHAM CJ. 1998. Smoke-induced seed germination in California chaparral. Ecology 79: 2320-2336.

KULKARNI MG; SPARG SG; VAN STADEN J. 2006a. Dark conditioning, cold stratification and a smoke-derived compound enhance the germination of Eucomis autumnalis subsp. autumnalis seeds. South African Journal of Botany 2: 157-162.

KULKARNI MG; SPARG SG; LIGHT ME; VAN STADEN J. 2006b. Stimulation of rice (Oryza sativa L.) seedling vigour by smoke-water and butenolide. J. Agronomy \& Crop Science 192: 395-398.

KULKARNI MG; STREET RA; VAN STADEN J. 2007a. Germination and seedling growth requirements for propagation of Dioscorea dregeana (Kunth) Dur. and Schinz - a tuberous medicinal plant. South African Journal of Botany 73: 131-137.

KULKARNI MG; ASCOUGH GD; VAN STADEN J. 2007b. Effects of foliar applications of smoke-water and a smokeisolated butenolide on seedling growth of okra and tomato. Hortscience 42: 179-182.

KULKARNI MG; SPARG SG; VAN STADEN J. 2007c. Germination and post-germination response of Acacia seeds to smoke-water and butenolide, a smoke-derived compound. Journal of Arid Environments 69: 177-187.

MERRITT DJ; KRISTIANSEN M; FLEMATTI GR; TURNER SR; GHISALBERTI EL; TRENGOVE RD; DIXON KW. 2006. Effects of a butenolide present in smoke on light-mediated germination of Australian Asteraceae. Seed Science Research 16: 29-35.

MINORSKY PV. 2000. Smoke-induced germination. Plant Physiology 128: 1167- 
1168.

ROCHE S; DIXON KW; PATE JS. 1998. For everything a season: smoke-induced seed germination and seedling recruitment in a Western Australian Banksia woodland. Australian Journal of Ecology 23: 111-120.

SPARG SG; KULKARNI MG; LIGHT ME; VAN STADEN J. 2005. Improving seedling vigour of indigenous medicinal plants with smoke. Bioresource Technology 96: 1323-1330.

SPARG SG; KULKARNI MG; VAN STADEN J. 2006. Aerosol smoke and smoke-water stimulation of seedling vigor of a commercial maize cultivar. Crop Science 46: 1336-1340.

STEVENS JC; MERRITT DJ; FLEMATTI GR; GHISALBERTI EL; DIXON KW. 2007. Seed germination of agricultural weeds is promoted by the butenolide 3 -methyl- $2 H$-furo[2,3-c] pyran-2-one under laboratory and field conditions. Plant Soil 298: 113-124.

VAN STADEN J; JÄGER AK; LIGHT ME; BURGER BV. 2004. Isolation of the major germination cue from plant-derived smoke. South African Journal of Botany 70: 654-659.
VAN STADEN J; SPARG SG; KULKARNI MG; LIGHT ME. 2006. Post-germination effects of the smoke-derived compound 3-methyl- $2 \mathrm{H}$ furo[2,3-c]pyran-2-one, and its potential as a preconditioning agent. Field Crops Research 98: 98-105.

VAN STADEN J; KULKARNI MG; ASCOUGH GD. 2007. The promotion of tomato and okra seedling growth by foliar applications of smoke-water and a smoke-isolated butenolide. South African Journal of Botany 73: 318. 